\title{
Proper scalar product for tachyon representations in configuration space
}

\author{
F. F. López-Ruiz $\oplus^{*}$ \\ Departamento de Física Aplicada, Universidad de Cádiz, \\ Campus de Puerto Real, E-11510 Puerto Real, Cádiz, Spain \\ J. Guerrero $\oplus^{\dagger, *}$ \\ Departamento de Matemáticas, Universidad de Jaén, Campus las Lagunillas, 23071 Jaén, Spain \\ V. Aldaya $\odot^{\S}$ \\ Instituto de Astrofísica de Andalucía (IAA-CSIC), Glorieta de la Astronomía, E-18080 Granada, Spain
}

(Received 30 July 2020; accepted 5 November 2020; published 1 December 2020)

\begin{abstract}
We propose a new inner product for scalar fields that are solutions of the Klein-Gordon equation with $m^{2}<0$. This inner product is nonlocal, bearing an integral kernel including Bessel functions of the second kind. The associated norm proves to be positive definite in the subspace of oscillatory solutions, as opposed to the conventional one, although some regularization procedure is required. Poincaré transformations are unitarily implemented on this subspace, which is the support of a unitary and irreducible representation of the proper orthochronous Poincaré group. We also provide a new Fourier transform between configuration and momentum spaces, which is unitary, and recover the projection onto the representation space. This new scenario suggests a revision of the corresponding quantum field theory.
\end{abstract}

DOI: 10.1103/PhysRevD.102.125010

\section{INTRODUCTION}

The Klein-Gordon equation with a negative squared mass, $m^{2}=-\kappa^{2}<0$, generated an interesting debate in the late 1960's and the 1970's. Assuming the usual signature $(+,-,-,-)$ as well as units in which $\hbar=c=1$, it is written

$$
\partial_{\mu} \partial^{\mu} \phi-\kappa^{2} \phi=0 .
$$

Originally, the corresponding quantum field theory was thought to represent faster-than-light particles [1]. However, the theory did not succeed; one of the main issues being the problems with the causality [2] and the unitary implementation of Poincaré transformations on this simple model [3-7]. In this sense, although the representations of the Poincare group for $m^{2}<0$ were not originally analyzed by Wigner [8], later authors constructed

*paco.lopezruiz@uca.es

†julio.guerrero@ujaen.es

${ }^{\sharp}$ Also at Institute Carlos I of Theoretical and Computational Physics, University of Granada, Fuentenueva s/n, 18071 Granada, Spain.

${ }^{\S}$ valdaya@iaa.es

Published by the American Physical Society under the terms of the Creative Commons Attribution 4.0 International license. Further distribution of this work must maintain attribution to the author(s) and the published article's title, journal citation, and DOI. Funded by SCOAP ${ }^{3}$. such representations, but only in momentum space [9-11] with support on the one-sheet hyperboloid. A unitary and irreducible representation realized on configuration space was still, surprisingly, lacking.

More recently, tachyonic modes were found to be present in many different physical models [12-22] and have been assumed to represent unstable states. Regardless of the nature of tachyonic modes, either fundamental or not, or their actual role in any physical theory, our aim in this paper is to point out that a description of the scalar solutions of (1) consistent with a unitary and irreducible representation of the Poincaré group cannot rely on the standard inner product,

$$
\langle\phi, \varphi\rangle_{\mathrm{st}}=i \int_{\Sigma} d \sigma^{\mu}\left(\phi(x)^{*} \partial_{\mu} \varphi(x)-\partial_{\mu} \phi(x)^{*} \varphi(x)\right),
$$

where $x=\left(x^{0}, \vec{x}\right), \Sigma$ is a spatial Cauchy hypersurface, and $d \sigma^{\mu}$ its surface element, but rather on the proposed inner product,

$$
\begin{aligned}
\langle\phi, \varphi\rangle= & \frac{\kappa^{2}}{4 \pi} \int_{\Sigma} d \sigma^{\mu} \int_{\Sigma^{\prime}} d \sigma^{\nu^{\prime}}\left(\phi(x)^{*} \partial_{\mu} \partial_{\nu^{\prime}} K\left(x-x^{\prime}\right) \varphi\left(x^{\prime}\right)\right. \\
& \left.+\partial_{\mu} \phi(x)^{*} K\left(x-x^{\prime}\right) \partial_{\nu^{\prime}} \varphi\left(x^{\prime}\right)\right),
\end{aligned}
$$

where $K(x)=-\frac{Y_{1}(\kappa s)}{\kappa s}$ with $s=\sqrt{\vec{x}^{2}-\left(x^{0}\right)^{2}}$, and $Y_{1}(x)$ is a Bessel function of the second kind. If we choose to fix $\Sigma$ at $x^{0}=0$, we obtain the more convenient form, 


$$
\begin{aligned}
\langle\phi, \varphi\rangle= & \frac{\kappa^{2}}{4 \pi} \int d^{3} x \int d^{3} x^{\prime}\left(\phi(x)^{*} k_{2}\left(\left|\vec{x}-\vec{x}^{\prime}\right|\right) \varphi\left(x^{\prime}\right)\right. \\
& \left.+\partial_{0} \phi(x)^{*} k_{1}\left(\left|\vec{x}-\vec{x}^{\prime}\right|\right) \partial_{0^{\prime}} \varphi\left(x^{\prime}\right)\right)
\end{aligned}
$$

where $k_{2}(r)=\frac{\tilde{Y}_{2}(\kappa r)}{r^{2}}$ and $k_{1}(r)=-\frac{Y_{1}(\kappa r)}{\kappa r}$. The tilde in $\tilde{Y}_{2}(x)$ indicates that a proper regularization is required (see later). Note that, even though the kernels $k_{2}(r)$ and $k_{1}(r)$ are oscillatory, they are positive-definite kernels on a suitable subspace (see below). With the aid of (3)-(4), we want to show that the quantum theory of a scalar free "tachyon" field with wave equation (1) is self-consistent and behaves as the scalar $m^{2}<0$ representation of the Poincaré group.

The motivation for (4) and its covariant version (3) is traced back to the pioneer study of nonlocal invariant inner products on spaces of solutions of the Klein-Gordon $\left(m^{2}>0\right)$ and Helmholtz equations given in Ref. [23]. There, the authors analyze general nonlocal inner products involving general integral kernels, the solutions, and their first-order derivatives. Imposing invariance conditions under the corresponding symmetry group, they recover, in particular, the inner product (2) for the usual $m^{2}>0$ Klein-Gordon solutions (invariance under Poincaré group forces the kernels to be Dirac delta functions in the case of the manifestly covariant representation, where both signs of the energy are contained). The proposed inner product (4) turns out to be a natural extension for $m^{2}<0$ of the analysis there realized, allowing for regularizable divergent kernels. Indeed, Poincaré invariance fixes the form and coefficients of the kernels $k_{1}$ and $k_{2}$ and their covariant version $K$.

\section{THE SPACE OF OSCILLATORY SOLUTIONS}

We define the Hilbert space of the theory $H_{\text {osc }}$ as the closure of the linear span of solutions of (1) made of exponentials with real frequencies, that is,

$\psi_{\vec{p} \pm}(x)=\frac{1}{\sqrt{2(2 \pi)^{3}}} e^{-i p_{\mu} x^{\mu}}, \quad p_{0}= \pm \omega_{p} \equiv \pm \sqrt{\vec{p}^{2}-\kappa^{2}}$,

where $|\vec{p}| \geq \kappa$ necessarily. Therefore, there is a gap in the norm of spatial momentum. This subspace of solutions will be called oscillatory solutions, since they represent solutions with an oscillatory behavior, in contrast with solutions with $|\vec{p}|<\kappa$ for which $p_{0}$ is imaginary, and therefore, they grow or decrease exponentially in time. The gap in spatial momentum also introduces a spatial oscillatory behavior. Note that this classification is Lorentz (and Poincaré) invariant, since a boost $p_{\mu} \rightarrow p_{\mu}^{\prime}$ does not change the relation, $p_{0}^{\prime 2}-\left|\vec{p}^{\prime}\right|^{2}+\kappa^{2}=0 \Rightarrow\left|\vec{p}^{\prime}\right|^{2}=\kappa^{2}+p_{0}^{\prime 2} \geq \kappa^{2}$.

The restriction to $|\vec{p}| \geq \kappa$ had already been noted by Feinberg in Ref. [1]. Now, mainly in view of (4), we highlight several properties singularizing that space. First, the initial value problem defined by (1), $\Sigma$, and the prescribed initial values $\phi(\vec{x})=\left.\phi(x)\right|_{\Sigma}$ and $\dot{\phi}(\vec{x})=$ $\left.\partial_{0} \phi(x)\right|_{\Sigma}$, is well-posed for oscillatory solutions; i.e., the solution's behavior changes continuously with properly given initial conditions, whereas it is ill-posed for real exponential solutions. We put emphasis on "properly" because $\phi(\vec{x})$ and $\dot{\phi}(\vec{x})$ cannot be arbitrary functions belonging to $L^{2}(\Sigma)$ : they must satisfy that their Fourier transforms $\hat{\phi}(\vec{p})$ and $\hat{\dot{\phi}}(\vec{p})$ have support on $|\vec{p}| \in[\kappa,+\infty)$. Second, the Poincaré infinitesimal generators $P_{\mu}=i \partial_{\mu}$ and $M_{\mu \nu}=x_{\mu} P_{\nu}-x_{\nu} P_{\mu}$ are Hermitian with respect to the inner product (4). In order to check this explicitly, some recurrence relations for the Bessel functions are required (see Ref. [24] for a similar computation). The inner product proves to be invariant under Poincaré transformations and, in particular, time invariant (the proof of this statement is parallel to that presented in Ref. [23] for the solutions of the Helmholtz equation and of the Klein-Gordon equation with $\left.m^{2}>0\right)$. This way, $\phi(x)$ and $\partial_{0} \phi(x)$ in (4) can be replaced by initial conditions on $\Sigma, \phi(\vec{x})$ and $\dot{\phi}(\vec{x})$.

Also, the representation of the proper orthochronous Poincaré group $\mathcal{P}_{+}^{\uparrow}$ on oscillatory solutions with the inner product (4), given by the exponentiation of $P_{\mu}$ and $M_{\mu \nu}$, is irreducible. That includes both positive and negative frequencies, in contrast with the $m^{2}>0$ case. A way to verify this is by introducing a unitary transformation [see Eqs. (8)-(10)] between the space of oscillatory solutions and the momentum space representation, where the unitary and irreducible representation for $m^{2}<0$ is known [10].

Finally, the inner product (4) turns out to define a positive definite norm on the space of oscillatory solutions, which is key to establish the equivalence between the configuration space representation and the known momentum space representation, with support on the one-sheet hyperboloid and which bears a positive definite inner product: the well-known expression (2) needs to be discarded for the configuration space representation as it fails to be positive definite.

\section{UNITARY EQUIVALENCE WITH MOMENTUM SPACE}

In the momentum space realization of the scalar representation [10], the Hilbert space $\mathcal{H}_{m}$ is given by functions on the one-sheet hyperboloid normalizable with respect to the integration measure $d^{4} \mu(p) \propto \delta\left(p^{2}+\kappa^{2}\right) d^{4} p$. The Cartesian projection on $p_{0}=0$ requires distinguishing a positive frequency component $\phi^{+}(\vec{p})$ and a negative frequency one $\phi^{-}(\vec{p})$, in such a way that $\phi(\vec{p})=$ $\left(\phi^{+}(\vec{p}), \phi^{-}(\vec{p})\right)$. The inner product is then given by

$$
\langle\phi, \varphi\rangle_{m}=\int_{|\vec{p}|>\kappa} \frac{d^{3} p}{\omega_{p}}\left(\phi^{+}(\vec{p})^{*} \varphi^{+}(\vec{p})+\phi^{-}(\vec{p})^{*} \varphi^{-}(\vec{p})\right) .
$$


The subspaces of positive and negative frequencies are orthogonal, but they are not invariant subspaces since Lorentz boosts mix them up. Note also that this inner product is nondegenerate on functions with support on $|\vec{p}|>\kappa$, in agreement with $\mathcal{H}_{m}$.

The momentum space counterpart of (5), eigenstates of momentum $\vec{p}^{\prime}$, are given by (recall that $\left|\vec{p}^{\prime}\right| \geq \kappa$ is required) $\psi_{\vec{p}^{\prime}+}(\vec{p})=\left(\omega_{p} \delta^{(3)}\left(\vec{p}-\vec{p}^{\prime}\right), 0\right)$ (for positive frequency states), and $\psi_{\vec{p}^{\prime}-}(\vec{p})=\left(0, \omega_{p} \delta^{(3)}\left(\vec{p}-\vec{p}^{\prime}\right)\right)$ (for negative frequency states). The scalar product among them gives

$$
\left\langle\psi_{\vec{p} \sigma}, \psi_{\vec{p}^{\prime} \sigma^{\prime}}\right\rangle_{m}=\delta_{\sigma \sigma^{\prime}} \omega_{p} \delta^{(3)}\left(\vec{p}-\vec{p}^{\prime}\right),
$$

where $\sigma, \sigma^{\prime}= \pm$.

Let us find the transformation taking momentum space wave functions $\left(\phi^{+}(\vec{p}), \phi^{-}(\vec{p})\right)$ to configuration space wave functions characterized by initial conditions $\phi(\vec{x})$ and $\dot{\phi}(\vec{x})$. Any oscillatory solution to (1) can be expanded in terms of (5),

$$
\phi(x)=\int_{|\vec{p}|>\kappa} \frac{d^{3} p}{\omega_{p}}\left(\phi^{+}(\vec{p}) \psi_{\vec{p}+}(x)+\phi^{-}(\vec{p}) \psi_{\vec{p}-}(x)\right) .
$$

Differentiating with respect to $x^{0}$ and making $x^{0}=0$ gives

$$
\phi(\vec{x})=\frac{1}{\sqrt{2(2 \pi)^{3}}} \int_{|\vec{p}|>\kappa} \frac{d^{3} p}{\omega_{p}}\left(\phi^{+}(\vec{p})+\phi^{-}(\vec{p})\right) e^{i \vec{p} \cdot \vec{x}},
$$

$$
\dot{\phi}(\vec{x})=\frac{-i}{\sqrt{2(2 \pi)^{3}}} \int_{|\vec{p}|>\kappa} d^{3} p\left(\phi^{+}(\vec{p})-\phi^{-}(\vec{p})\right) e^{i \vec{p} \cdot \vec{x}}
$$

Let us now define the following inverse transformation:

$$
\phi^{ \pm}(\vec{p})=\frac{1}{\sqrt{2(2 \pi)^{3}}} \int d^{3} x\left(\left(\omega_{p}\right)_{+} \phi(\vec{x}) \pm i\left(\omega_{p}\right)_{+}^{0} \dot{\phi}(\vec{x})\right) e^{-i \vec{p} \cdot \vec{x}}
$$

where $\left(\omega_{p}\right)_{+}=\omega_{p}$ for $|\vec{p}| \geq \kappa,\left(\omega_{p}\right)_{+}=0$ for $|\vec{p}|<\kappa$, $\left(\omega_{p}\right)_{+}^{0}=1$ for $|\vec{p}| \geq \kappa$, and $\left(\omega_{p}\right)_{+}^{0}=0$ for $|\vec{p}|<\kappa$. Equation (10) together with (8) and (9) define a "Fourier" transform and its inverse, relating configuration and momentum spaces. In particular, it can be checked that eigenstates of momentum $\vec{p}^{\prime}$ in configuration space are mapped into eigenstates of momentum $\vec{p}^{\prime}$ in momentum space and vice versa.

We can now show that both configuration and momentum space representations on $\mathcal{H}_{\text {osc }}$ and $\mathcal{H}_{m}$, respectively, are unitarily equivalent. Given that $\psi_{\vec{p} \sigma}(\vec{x})=\frac{1}{\sqrt{2(2 \pi)^{3}}} e^{i \vec{p} \cdot \vec{x}}$ and $\dot{\psi}_{\vec{p} \sigma}(\vec{x})=\frac{-i \sigma \omega_{p}}{\sqrt{2(2 \pi)^{3}}} e^{i \vec{p} \cdot \vec{x}}$, with $\sigma= \pm$, let us compute (7) in configuration space using (4) (we insert $e^{i \vec{p} \cdot \vec{x}^{\prime}} e^{-i \vec{p} \cdot \vec{x}^{\prime}}$ when necessary),

$$
\begin{aligned}
\left\langle\psi_{\vec{p} \sigma}, \psi_{\vec{p}^{\prime} \sigma^{\prime}}\right\rangle & =\frac{\kappa^{2}}{4 \pi} \frac{1}{2(2 \pi)^{3}} \int d^{3} x \int d^{3} x^{\prime}\left(e^{-i \vec{p} \cdot \vec{x}} k_{2}\left(\left|\vec{x}-\vec{x}^{\prime}\right|\right) e^{i \vec{p}^{\prime} \cdot \vec{x}^{\prime}}+i \sigma \omega_{p} e^{-i \vec{p} \cdot \vec{x}} k_{1}\left(\left|\vec{x}-\vec{x}^{\prime}\right|\right)\left(-i \sigma^{\prime} \omega_{p^{\prime}} e^{i \vec{p}^{\prime} \cdot \vec{x}^{\prime}}\right)\right) \\
& =\frac{\kappa^{2}}{4(2 \pi)^{4}} \int d^{3} x^{\prime}\left\{\int d^{3} x k_{2}\left(\left|\vec{x}-\vec{x}^{\prime}\right|\right) e^{-i \vec{p} \cdot\left(\vec{x}-\vec{x}^{\prime}\right)}+\sigma \sigma^{\prime} \omega_{p} \omega_{p^{\prime}} \int d^{3} x k_{1}\left(\left|\vec{x}-\vec{x}^{\prime}\right|\right) e^{-i \vec{p} \cdot\left(\vec{x}-\vec{x}^{\prime}\right)}\right\} e^{-i\left(\vec{p}-\vec{p}^{\prime}\right) \cdot \vec{x}^{\prime}} \\
& =\frac{1}{2(2 \pi)^{3}} \int d^{3} x^{\prime}\left\{\left(\omega_{p}\right)_{+}+\sigma \sigma^{\prime} \omega_{p^{\prime}}\left(\omega_{p}\right)_{+}^{0}\right\} e^{-i\left(\vec{p}-\vec{p}^{\prime}\right) \cdot \vec{x}^{\prime}}=\frac{1}{2}\left(1+\sigma \sigma^{\prime}\right)\left(\omega_{p}\right)_{+} \delta^{(3)}\left(\vec{p}-\vec{p}^{\prime}\right)=\delta_{\sigma \sigma^{\prime}}\left(\omega_{p}\right)_{+} \delta^{(3)}\left(\vec{p}-\vec{p}^{\prime}\right) .
\end{aligned}
$$

Again, $\left(\omega_{p}\right)_{+}$and $\left(\omega_{p}\right)_{+}^{0}$ are zero if $|\vec{p}|<\kappa$. We have used that $\int d^{3} x e^{-i \vec{p} \cdot \vec{x}}=(2 \pi)^{3} \delta^{(3)}(\vec{p})$ and that the integral involving the second kernel is $\int d^{3} x k_{1}(|\vec{x}|) e^{-i \vec{p} \cdot \vec{x}}=\frac{4 \pi}{\kappa^{2}} \frac{\left(\omega_{p}\right)_{+}^{0}}{\omega_{p}}$. The integral involving the first kernel is divergent. However, sense can be made of this integral with an adequate regularization. For instance, with dimensional regularization in dimension $d$, analytical continuation to $d=3$ provides $\int_{\mathbb{R}^{3}} d^{3} x k_{2}(|\vec{x}|) e^{-i \vec{p} \cdot \vec{x}}=\frac{4 \pi}{\kappa^{2}}\left(\omega_{p}\right)_{+} . \quad$ That shows the positive definiteness of the kernels of the proposed inner product.

With all that, we verify the unitary equivalence of configuration space and momentum space representations: we see that the computation above is in agreement with (7) for $|\vec{p}| \geq \kappa$, which is satisfied within the Hilbert space. This also confirms the fact that the inner product is nondegenerate only for functions whose Fourier transform have support on $|\vec{p}|>\kappa$, that is, for oscillatory solutions of (1), exactly like (6).

We should remark that using the usual scalar product (2) would lead, on the contrary, to $\left\langle\psi_{\vec{p} \sigma}, \psi_{\vec{p}^{\prime} \sigma^{\prime}}\right\rangle=$ $\frac{1}{2}\left(\sigma+\sigma^{\prime}\right) \omega_{p} \delta^{(3)}\left(\vec{p}-\vec{p}^{\prime}\right)$, which is in clear conflict with the positive definiteness of the inner product (6) in momentum space representation and the restriction to the space of oscillatory solutions in configuration space representation.

We can now work out an example. Consider the isotropic states defined in momentum space to have compact 


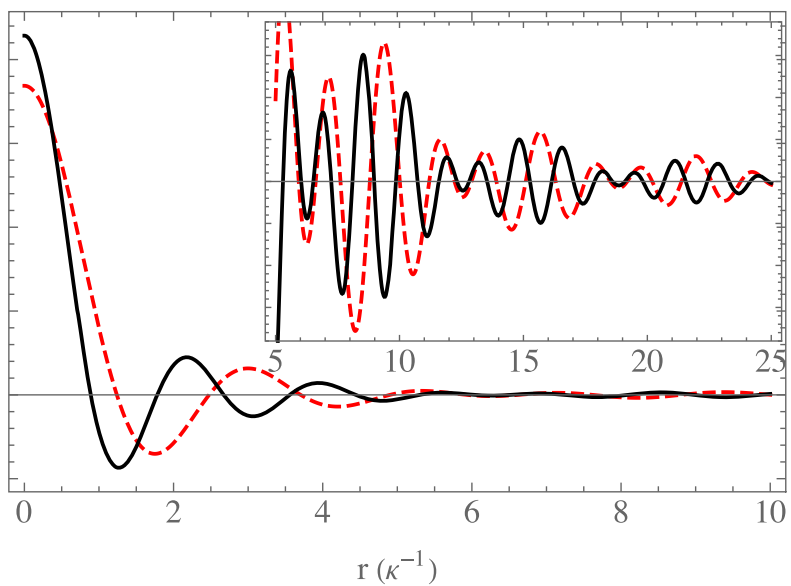

FIG. 1. Configuration space radial wave functions $\varphi_{p_{a} p_{b}}(r)$ of two orthogonal band-limited symmetric states with the momentum space wave function $\varphi_{p_{a} p_{b}}^{ \pm}(\vec{p})=N \omega_{p} \theta\left(|\vec{p}|-p_{a}\right) \theta\left(p_{b}-|\vec{p}|\right)$. The radial coordinate $r$ is in units of $\kappa^{-1}$. The dashed red line represents the state with $p_{a}=2 \kappa, p_{b}=3 \kappa$, and the solid black represents $p_{a}=3 \kappa, p_{b}=4 \kappa$. A magnification over a wider domain is shown.

support: $\varphi_{p_{a} p_{b}}^{ \pm}(\vec{p})=N \omega_{p} \theta\left(|\vec{p}|-p_{a}\right) \theta\left(p_{b}-|\vec{p}|\right)$, where $\theta(x)$ is the Heaviside step function, $N$ a normalizing constant, and $p_{b}-p_{a}$ is the bandwidth $\left(p_{b}>p_{a}>\kappa>0\right)$. We take symmetric states with $\varphi_{p_{a} p_{b}}^{+}(\vec{p})=\varphi_{p_{a} p_{b}}^{-}(\vec{p})$ for simplicity, as this implies $\dot{\varphi}_{p_{a} p_{b}}(\vec{x})=0$ [see (9)], whereas using (8), we find $\varphi_{p_{a} p_{b}}(\vec{x})=\sqrt{2} N\left(\left(\frac{p_{b}}{\mid \vec{x}}\right)^{\frac{3}{2}} J_{\frac{3}{2}}\left(p_{b}|\vec{x}|\right)-\left(\frac{p_{a}}{\mid \vec{x}}\right)^{\frac{3}{2}} J_{\frac{3}{2}}\left(p_{a}|\vec{x}|\right)\right)$, where $J_{n}(x)$ is a Bessel function of the first kind. Two of such states with nonoverlapping bandwidths are obviously orthogonal in view of (6). In configuration space this is not obvious given that the inner product (4) is nonlocal. See Fig. 1 for an illustration. The scalar product (2) always yields $\left\langle\varphi_{p_{a} p_{b}}, \varphi_{p_{c} p_{d}}\right\rangle_{s t}=0$ for all those band-limited symmetric states and therefore does not reproduce the Hilbert space structure of $\mathcal{H}_{m}$, which is only possible due to the nonlocal nature of (4).

\section{PROJECTION ON THE HILBERT SPACE}

Let us show another manifestation of the fact that initial conditions for (1) can not be arbitrary functions on $\Sigma$ if the corresponding solutions $\phi(x)$ are intended to support a unitary and irreducible representation of $\mathcal{P}_{+}^{\uparrow}$ (we now deepen in some ideas already present in Ref. [1]). Inserting (8) and (9) in (10), we obtain $\left(\omega_{p}\right)_{+}^{0} \phi^{ \pm}(\vec{p}) \equiv$ $\tilde{\phi}^{ \pm}(\vec{p})$; that is, the identity as long as $\phi^{ \pm}(\vec{p})$ has support on $|\vec{p}| \geq \kappa$ only. Now, if we use (10) in (8), we get

$$
\begin{aligned}
& \frac{1}{2(2 \pi)^{3}} \int_{|\vec{p}|>\kappa} \frac{d^{3} p}{\omega_{p}}\left\{\int d^{3} x^{\prime}\left(\left(\omega_{p}\right)_{+} \phi\left(\vec{x}^{\prime}\right)+i\left(\omega_{p}\right)_{+}^{0} \dot{\phi}\left(\vec{x}^{\prime}\right)\right)+\int d^{3} x^{\prime}\left(\left(\omega_{p}\right)_{+} \phi\left(\vec{x}^{\prime}\right)-i\left(\omega_{p}\right)_{+}^{0} \dot{\phi}\left(\vec{x}^{\prime}\right)\right)\right\} e^{i \vec{p} \cdot\left(\vec{x}-\vec{x}^{\prime}\right)} \\
& =\frac{1}{2(2 \pi)^{3}} \int d^{3} x^{\prime}\left\{\int_{|\vec{p}|>\kappa} d^{3} p 2\left(\omega_{p}\right)_{+}^{0} e^{i \vec{p} \cdot\left(\vec{x}-\vec{x}^{\prime}\right)}\right\} \phi\left(\vec{x}^{\prime}\right) \\
& =\phi(\vec{x})-\int d^{3} x^{\prime}\left(\frac{\kappa}{2 \pi}\right)^{\frac{3}{2}} \frac{J_{\frac{3}{2}}\left(\kappa\left|\vec{x}-\vec{x}^{\prime}\right|\right)}{\left|\vec{x}-\vec{x}^{\prime}\right|^{\frac{3}{2}}} \phi\left(\vec{x}^{\prime}\right) \equiv \tilde{\phi}(\vec{x}),
\end{aligned}
$$

where we have written the integral as $\int_{|\vec{p}|>\kappa}=\int_{\mathbb{R}^{3}}-\int_{|\vec{p}|<\kappa}$, the first term giving a Dirac delta, and the second one the kernel $k_{3 / 2}(r)=\left(\frac{\kappa}{2 \pi}\right)^{\frac{3}{2}} \frac{J_{\frac{3}{3}}(r)}{r^{3 / 2}}$, with a Bessel function of the first kind. An analogous result is obtained for $\dot{\phi}(\vec{x})$.

That may look puzzling at first sight: the expected result would be the identity. However, it must be noted that the convolution with the kernel $k_{3 / 2}(r)$ is zero for those functions $\phi(\vec{x})$ and $\dot{\phi}(\vec{x})$ whose Fourier transforms $\hat{\phi}(\vec{p})$ and $\hat{\dot{\phi}}(\vec{p})$ have support on $|\vec{p}| \in[\kappa,+\infty)$. Therefore, the convolution with the kernel $\delta^{(3)}\left(\vec{x}-\vec{x}^{\prime}\right)-k_{3 / 2}\left(\kappa\left|\vec{x}-\vec{x}^{\prime}\right|\right)$ projects onto the Hilbert space $\mathcal{H}_{\mathrm{osc}}$. As a consequence, within the Hilbert space, supporting the $m^{2}<0$ irreducible representation of $\mathcal{P}_{+}^{\uparrow}$, the composition of (8)-(10) is indeed the identity, as expected.

A further comment is in order: the convolution operator with the kernel $\delta^{(3)}\left(\vec{x}-\vec{x}^{\prime}\right)-k_{3 / 2}\left(\kappa\left|\vec{x}-\vec{x}^{\prime}\right|\right)$ is a nontrivial operator in $L^{2}\left(\mathbb{R}^{3}\right)$ which commutes with the operators of the Poincare group. However, it becomes the identity when we restrict to the irreducible representation on $\mathcal{H}_{\text {osc }}$, a fact compatible with Schur's lemma.

Let us check all that with an example. As initial conditions for (1), one might consider familiar Gaussian wave packets. For simplicity, we focus on a spherically symmetric initial state $\phi(\vec{x})=\frac{C}{(2 \pi)^{3 / 2} \sigma^{3}} \exp \left(-\frac{|\vec{x}|^{2}}{2 \sigma^{2}}\right)$ [equivalent results are obtained for Gaussian $\dot{\phi}(\vec{x})$, although we are not interested in the subsequent time evolution]. The result of the projection operation on the Hilbert space can be seen in Fig. 2: we observe that the well-localized Gaussian wave function acquires an oscillatory tail that decays slower than the original one; actually, $\tilde{\phi}(\vec{x}) \sim \frac{\cos (\kappa|\vec{x}|)}{|\vec{x}|^{2}}$, while $\phi(\vec{x}) \sim$ $\exp \left(-\frac{|\vec{x}|^{2}}{2 \sigma^{2}}\right)$. This turns out to be a general fact: no initial conditions for (1) localized in space can lead to oscillatory solutions; any $\phi(\vec{x})$ and $\dot{\phi}(\vec{x})$ are necessarily spread over the entire Cauchy hypersurface $\Sigma[1]$. 


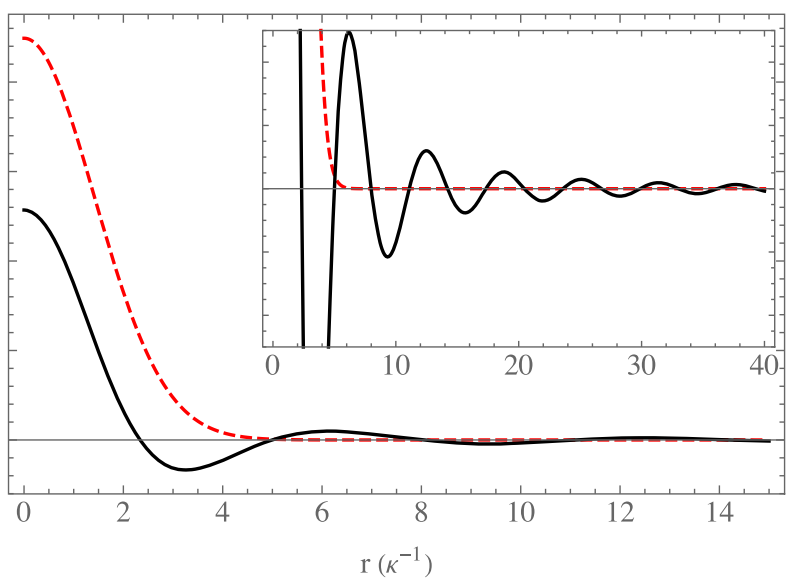

FIG. 2. The nonadmissible Gaussian wave function $\phi(r)$ as an initial condition (dashed red) and its corresponding (admissible) projection $\tilde{\phi}(r)$ on the Hilbert space of oscillatory solutions for $\sigma=2 / \kappa$ (solid black). The radial coordinate $r$ is in units of $\kappa^{-1}$. A magnification over a wider domain is shown to appreciate the oscillatory tail.

\section{DISCUSSION}

Once the missing scalar $m^{2}<0$ representation in configuration space have been realized, and the corresponding inner product established, there are still several issues to be addressed. Given that the inner product (4) [as well as (3)] is positive definite, it seems possible to define a relativistic quantum mechanics for wave functions satisfying (1). In fact, we have already seen that a suitable definition of the Hilbert space in terms of oscillatory, delocalized functions is necessary, as well as a nonlocal inner product. If possible, a precise definition of the density of probability in configuration space would also be required, a definition that might include a nonlocal operation, such as the convolution with certain oscillatory kernels (for the case of the Klein-Gordon equation with $m^{2}>0$, see Ref. [10]; see also Ref. [25]). However, it must be pointed out that these circumstances are not that new: we have already encountered an analogous situation for the quantum mechanics in momentum space of a free particle moving on a sphere [24], which turns out to be completely equivalent to the usual one in configuration space [26]. Of course, whether the delocalization of the wave function implies that the field is physically delocalized in space, raising the question of the existence of a particlelike behavior, requires further study, as we have already remarked that a probabilistic interpretation in accordance with the inner product (4) is to be developed. In any case, these peculiar features of the free theory may have implications in the analysis and even in the very definition of causality for an interacting "tachyonlike" quantum field theory.

Besides the already mentioned probabilistic interpretation, the nonscalar, infinite-dimensional representations [9] remain to be found in configuration space. To determine whether a scalar or nonscalar tachyonlike representation are to bear some physical significance at the fundamental level and/or fully develop a quantum field theory, a careful analysis of causality is required in light of the delocalization property. For that aim, the form of those interactions compatible with Poincare symmetry should also be analyzed, as well as the time evolution.

It would also be convenient to have at hand a generalized form of the present inner product in such a way that the same expression would be directly applicable to physical systems containing fields with a different sign of $m^{2}$, hence treating those fields on an equal footing in a unified manner within the theory. It seems straightforward to achieve this unification by following the scheme given in [23] and writing general inner products of the form,

$$
\langle\phi, \varphi\rangle=\int d^{3} x \int d^{3} x^{\prime}\left(\phi(x)^{*}, \partial_{0} \phi(x)^{*}\right)\left(\begin{array}{cc}
K_{11}\left(\left|\vec{x}-\vec{x}^{\prime}\right|\right) & K_{12}\left(\left|\vec{x}-\vec{x}^{\prime}\right|\right) \\
K_{21}\left(\left|\vec{x}-\vec{x}^{\prime}\right|\right) & K_{22}\left(\left|\vec{x}-\vec{x}^{\prime}\right|\right)
\end{array}\right)\left(\begin{array}{c}
\varphi\left(x^{\prime}\right) \\
\partial_{0^{\prime}} \varphi\left(x^{\prime}\right)
\end{array}\right) .
$$

Invariance under the corresponding representation of the Poincaré group will fix the values of the matrix kernel coefficients. For instance, for $m^{2}<0$, we recover Eq. (4) with $K_{12}=K_{21}=0$ and $K_{11}(r) \propto k_{2}(r)$ and $K_{22}(r) \propto k_{1}(r)$, with $k_{1}$ and $k_{2}$ given after Eq. (4). For $m^{2}>0$ and the manifestly covariant case (i.e., with both signs of the energy included), we recover Eq. (2) with $K_{11}=K_{22}=0$ and $K_{12}(r) \propto \delta(r)$ and $K_{21}(r) \propto-\delta(r)$. Nevertheless, the inclusion of the $m^{2}=0$ case (and the $m^{2}>0$ case with only the positive sign of the energy, thus irreducible) requires further study.

Finally, the framework described here may play some role as a piece in a broader physical theory. There have been many contributions in that direction, such as in string theory $[12,13]$, supersymmetry [14], quantum field theory in curved spacetime [15], cosmology [16,17], spontaneous symmetry breaking $[18,19]$, QCD [20], condensed matter [21], or even in the study of liquids [22]. Maybe the analysis of quantum effects in those contexts might require a revision with the new inner product (4) (or a generalization) in mind. In fact, we want to stress that the aim of this work is more preliminary and/or fundamental: we have just provided the inner product (3)-(4) and, from that, the new configuration space realization of the scalar $m^{2}<0$ representation of $\mathcal{P}_{+}^{\uparrow}$. 


\section{ACKNOWLEDGMENTS}

The authors are very grateful to E. R. Arriola for fruitful discussions. We thank the Spanish Ministerio de Ciencia e Innovación (MICINN) for financial support (Grant No. FIS2017-84440-C2-2-P) and V. A. acknowledges financial support from the State Agency for Research of the Spanish MICINN through the "Center of Excellence Severo Ochoa" grant for the Instituto de Astrofísica de Andalucía (Grant No. SEV-2017-0709). J. G. acknowledges financial support from the Spanish MICINN (Grant No. PGC2018-097831-B-I00).
[1] G. Feinberg, Phys. Rev. 159, 1089 (1967).

[2] Y. Aharonov, A. Komar, and L. Susskind, Phys. Rev. 182, 1400 (1969).

[3] M. E. Arons and E. C. G. Sudarshan, Phys. Rev. 173, 1622 (1968).

[4] J. Dhar and E.C. G. Sudarshan, Phys. Rev. 174, 1808 (1968).

[5] B. Schroer, Phys. Rev. D 3, 1764 (1971).

[6] C. Jue, Phys. Rev. D 8, 1757 (1973).

[7] G. Feinberg, Phys. Rev. D 17, 1651 (1978).

[8] E. P. Wigner, Ann. Math. 40, 149 (1939).

[9] H. E. Moses, J. Math. Phys. (N.Y.) 9, 2039 (1968).

[10] L. Fonda and G. C. Ghirardi, Symmetry Principles in Quantum Physics (Marcel Dekker, New York, 1970), Chap. 5.

[11] N. Mukunda, Ann. Phys. (N.Y.) 61, 329 (1970).

[12] A. Sen, J. High Energy Phys. 08 (1998) 012.

[13] A. Armoni and E. Lopez, Nucl. Phys. B632, 240 (2002).

[14] J. Ellis, J. Giedt, O. Lebedev, K. Olive, and M. Srednicki, Phys. Rev. D 78, 075006 (2008).

[15] A. G. S. Landulfo, W. C. C. Lima, G. E. A. Matsas, and D. A. T. Vanzella, Phys. Rev. D 86, 104025 (2012).
[16] J. S. Bagla, H. K. Jassal, and T. Padmanabhan, Phys. Rev. D 67, 063504 (2003).

[17] E. M. Teixeira, A. Nunes, and N. J. Nunes, Phys. Rev. D 100, 043539 (2019).

[18] G. Felder, J. Garcia-Bellido, P. B. Greene, L. Kofman, A. Linde, and I. Tkachev, Phys. Rev. Lett. 87, 011601 (2001).

[19] G. Felder, L. Kofman, and A. Linde, Phys. Rev. D 64, 123517 (2001).

[20] T. Yokota, T. Kunihiro, and K. Morita, Phys. Rev. D 96, 074028 (2017).

[21] D. O. Oriekhov and L. S. Levitov, Phys. Rev. B 101, 245136 (2020).

[22] C. Yang, M. T. Dove, V. V. Brazhkin, and K. Trachenko, Phys. Rev. Lett. 118, 215502 (2017).

[23] S. Steinberg and K. B. Wolf, J. Math. Phys. (N.Y.) 22, 1660 (1981).

[24] J. Guerrero, F. F. López-Ruiz, and V. Aldaya, J. Phys. A 53, 145301 (2020).

[25] A. Mostafazadeh and F. Zamani, Ann. Phys. (Amsterdam) 321, 2183 (2006).

[26] V. Aldaya, J. Guerrero, F. F. López-Ruiz, and F. Cossío, J. Phys. A 49, 505201 (2016). 